(>)

\title{
LA INTERPRETACIÓN DE GAUTHIER DEL ARGUMENTO POLÍTICO HOBBESIANO
}

Oswaldo Plata Pineda 


\title{
LA INTERPRETACIÓN DE GAUTHIER DEL ARGUMENTO POLÍTICO HOBBESIANO*
}

\begin{abstract}
Resumen: David Gauthier interpreta el conflicto en el estado de naturaleza hobbesiano como un conflicto de orden racional surgido de la necesidad de los hombres de preservar la integridad e incrementar el bienestar individual. Desde su punto de vista, la solución hobbesiana a este conflicto plantea un esquema moral -con asiento racional- que permite compatibilizar las expectativas individuales y colectivas. En este estudio reconstruyo los argumentos que ofrece Gautier para sostener la tesis de que el contenido moral de las leyes de la naturaleza, presupuesto básico del contrato social, permite compatibilizar las expectativas individuales y colectivas.
\end{abstract}

Palabras clave: Hobbes, Gauthier, Moral, Razón, Convención.

\section{THE INTERPRETATION OF GAUTHIER OF HOBBESIAN POLITICAL ARGUMENT}

\begin{abstract}
David Gauthier interprets the conflict in the Hobbesian state of nature as a rational conflict arisen from the need of men to preserve their integrity and to increase their individual welfare. From his point of view, the Hobbesian solution to this conflict exposes a moral scheme -with a rational support- allowing the compatibility between individual and collective expectations. In this study I present the arguments that Gauthier offers to defend the idea that the Hobbesian moral theory premised on the dynamics of social contract reconciles individual and collective expectations.
\end{abstract}

Keywords: Hobbes, Gauthier, Moral, Reason, Convention.

Fecha de recepción: abril 6 de 2015

Fecha de aceptación: septiembre 9 de 2015

Forma de citar: Pineda, O. (2015). "La interpretación de Gauthier del argumento político hobbesiano". Revista Filosofía UIS. 15 (2). pp. 83-95.

Oswaldo Plata Pineda: Colombiano. Licenciado y Magister en Filosofía de la Universidad del Valle. Candidato a Doctor en Filosofía de la Universidad Pontificia Bolivariana. Docente de tiempo completo del Politécnico Colombiano Jaime Isaza Cadavid.

Correo electrónico: oswaldoplata10@gmail.com

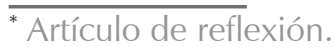




\section{LA INTERPRETACIÓN DE GAUTHIER DEL ARGUMENTO POLÍTICO HOBBESIANO}

En Logic of Leviathan (1969), Morals by Agreement (1987) y diversos artículos, David Gauthier examina la teoría hobbesiana de la política en orden a establecer un esquema moral — con asiento racional— que compatibilice las expectativas individuales y colectivas. Gauthier considera que, con base en Hobbes, se puede defender la tesis de que la racionalidad (entendida ella como la maximización del interés propio vía la selección de los mejores medios para la consecución de un fin específico) permite deducir del interés personal un tipo distintivo de moral restrictiva de los intereses individuales. Contradiciendo una larga tradición interpretativa que identifica a Hobbes como el campeón del egoísmo psicológico, Gauthier sostiene que es posible establecer una dinámica cooperativa en un escenario en el que el beneficio de los hombres (egoístas racionales) está en juego a partir de lo que todos y cada uno de ellos realiza. En orden a sostener lo anterior, Gauthier interpreta el conflicto en el estado de naturaleza hobbesiano como un conflicto de orden racional surgido de la necesidad de los hombres de preservar la integridad e incrementar el bienestar individual.

En el presente estudio expongo los argumentos que ofrece Gauthier para defender la idea de que la teoría hobbesiana de la moral presupuesta en la dinámica del contrato social compatibiliza las expectativas individuales y colectivas. En la primera parte de este estudio, señalo por qué Gauthier considera que con la introducción de un convencionalismo dual (Razón natural-comportamiento natural/Razón convencional-moral convencional) se compatibilizan las expectativas individuales y colectivas. Describo aquí la concepción hobbesiana de la razón (instrumentalista, subjetiva, derivada del derecho natural y proclive al error) y las consecuencias adversas que ella trae para la convivencia en el estado de naturaleza (situación de guerra de todos contra todos). En la segunda parte, presento la manera como la introducción de la noción de ley natural en el argumento político hobbesiano posibilita la superación de la condición miserable del estado de naturaleza y la institución de una moral convencional, un árbitro moral de las disputas y un conjunto de comportamientos sugerido por las leyes de la naturaleza. En la tercera y última parte, refiero el análisis que Gauthier realiza del problema de estabilidad de las convenciones (morales) del estado político. 
Centro aquí la atención en la respuesta que da Hobbes al caso del insensato y que Gauthier utiliza para apoyar su tesis de que la teoría hobbesiana de la política suministra un esquema moral que permite compatibilizar las expectativas individuales y colectivas.

I. De acuerdo con Gauthier, la teoría política de Hobbes no sólo contribuyó a la configuración del estado político modernosino, además, sentó las bases conceptuales de la teoría moral moderna1. "That Thomas Hobbes is the greatest of English political philosophers is a commonplace claim. That he is the greatest of English moral philosophers is not a commonplace. But it is true" (Gauthier, 1979 , p. 548). En efecto, Gauthier estima que la teoría hobbesiana del contrato social suministra un esquema moral — con asiento racional- que compatibiliza las expectativas individuales y colectivas. El contrato social hobbesiano efectúa esta compatibilización ilustrando cómo la racionalidad (entendida ella como la maximización del interés propio vía la selección de los mejores medios para la consecución de un fin específico) permite deducir del interés personal un tipo distintivo de moral restrictiva de los intereses individuales.

The morality that Hobbes establishes is minimal; it represents the weakest of constraints on natural maximizing behavior -that set by considerations of mutual advantage. It is only because each person has an interest in peace that each has grounds to accept the conventional reason and morality which together override the straightforward maximization of subjective value.

[...] Hobbes shows us that moral and social relationships are possible among persons in contexts in which they take no interest in one another's interests. Properly understood, this is one of the great liberating insights on which a free and democratic society is based (Gauthier, 1979, pp. 558-559).

Desde este punto de vista, el estado de naturaleza describe un escenario de negociación racional en el que las partes buscan la propia preservación y el incremento del bienestar propio. Para Gauthier, tal descripción transmite un convencionalismo dual (razón natural-comportamiento natural/razón convencional-moral convencional) que posibilita la superación de la condición miserable del estado de naturaleza:

\footnotetext{
${ }^{1}$ De acuerdo con Gauthier, el problema la teoría moral moderna es establecido por normas que se derivan de la economía: la utilidad, la racionalización como maximización y la idea de que los intereses no son "tuistic". La teoría moral moderna defiende la posibilidad de la moralidad en relación con estos tres dogmas. Gauthier considera que con la introducción de un convencionalismo dual (Razón natural-comportamiento natural /Razón convencionalmoral convencional) Hobbes instituyó los núcleos problémicos de la teoría moral moderna. En el artículo en comento, "Thomas Hobbes: Moral Theorist", Gauthier expone la manera como Hobbes desarrolla, sin saberlo, los tres dogmas antedichos.
} 


\begin{abstract}
The majority of moral theorists have, of course, sought to establish the possibility of morality by rejecting one or more of the economists' suppositions. They have offered alternative accounts of value, or reason, or interest. But the dogmas remain, and the bolder course is to embrace them. This is what Hobbes does, establishing a place for morality as a conventional constraint on our natural behavior. The tour de force in his theory is the reconciliation of maximizing rationality with constraining morality. How can one be rational in accepting the constraints of the laws of nature, and so not exercising one's full right of nature? The answer requires Hobbes's account of right reason. For his true moral theory is a dual conventionalism, in which a conventional reason, superseding natural reason, justifies a conventional morality, constraining natural behavior. And this dual conventionalism is Hobbes's enduring contribution to moral theory (Gauthier, 1979, p. 547).
\end{abstract}

La tesis de Gauthier es que la definición hobbesiana de razón transmite simultáneamente la idea de convencionalismo (recta razón) y la consideración de que los hombres sólo razonan sobre lo particular ("based on desires and values" (Gauthier, 1979, pp. 548-549)), sugiriendo con ello que la razón es autorreferencial y se encuentra conectada con el incremento del propio bienestar. En El Leviatán, Hobbes define, en efecto, la razón como un calcular, un sumar y restar consecuencias: "[...] la Razón no es otra cosa que un calcular, es decir, un sumar y restar consecuencias de los nombres universales que hemos convenido para marcar y siginificar nuestros pensamientos" (Hobbes, 1994, p. 46). Esta definición llevada al plano concreto significa que el hombre persigue sus fines valiéndose de los distintos medios de que dispone. Tal prosecución comprende un proceso cognitivo más o menos constante: primero, la definición del objetivo de su deseo; segundo, el cálculo de los medios necesarios para alcanzarlo; y tercero, la consideración de los intereses de los otros agentes. Los tres pasos del proceso son realizados por el hombre en todas las circunstancias, pero es, para Gauthier, el tercero el que define distintivamente la estructura psicológica del hombre. Pues, al estar ligado al incesante deseo de poder $^{2}$ (a saber, tendencia humana no solo a satisfacer las necesidades vitales que brotan de los deseos, sino a prevalecer sobre los poderes de los otros) y al carácter subjetivo de los juicios morales ("[...] las palabras de bueno, malo y desdeñable siempre son utilizadas en relación a la persona que las usa, ya que no hay nada que sea simple y absolutamente ninguna de las tres cosas" ( Hobbes, 1994, p. 55)), explica por qué los hombres persiguen estratégicamente los fines trazados sin observar ningún tipo de norma moral ni considerar los perjuicios que su acción genere, a no ser que hacerlo los beneficie manifiestamente. Gauthier estima que las condiciones del estado de naturaleza ilustran de manera precisa la forma cómo funciona la racionalidad instrumental de los hombres y cómo ella deviene en anarquía: "para lograr su fin,

\footnotetext{
${ }^{2}$ El deseo de poder, que "solo cesa con la muerte", es capital en el argumento político, porque él explica el surgimiento del conflicto en el estado de naturaleza. De hecho, el poder es un concepto comparativo que hace que el valor de las cosas y las personas dependa de un agente externo.
} 
que es, principalmente, su propia conservación y, algunas veces, solo su deleite, [los hombres] se empeñan en destruirse y someterse mutuamente" (Hobbes, 1994, p. 114).

Gauthier reconoce que Hobbes parte del supuesto ${ }^{3}$ de que los hombres, en tanto que seres racionales, saldrán del estado de naturaleza e instituirán el estado político ("posibilidad que, en parte, radica en sus pasiones y, en parte, en su razón". Hobbes, 1994, p. 117). Pese a esto, Gauthier afirma que el objetivo de Hobbes con la formulación del estado hipotético del estado de naturaleza no es otro que demostrar la necesidad teórica de una estructura coercitiva que ponga fin a la guerra e instaure la paz:

But of course Hobbes supposes that persons can exit from the state of nature, and his account of conflict is intended to establish, not that rational persons would face universal war, but that they would accept an institutional structure that provides the coercive force needed to motivate compliance with the laws of nature, which Hobbes describes as "convenient Articles of Peace, upon which men may be drawn to agreement", and so to establish and maintain peaceable cooperation (1979, p. 72).

Las condiciones impuestas por Hobbes al estado de naturaleza crean un escenario de combate mortal entre los hombres, debido a que todos desean preservar su existencia asegurando su bienestar y todos gozan de un derecho ilimitado a todas las cosas. Esta generalización es particularmente relevante, afirma Gauthier, porque el argumento hobbesiano plantea que todos los hombres creen que son, en términos generales, iguales o, en otras palabras, que las desigualdades de cuerpo y de alma no marcan una diferencia sustantiva entre ellos y, en consecuencia, es legítimo albergar la esperanza de alcanzar los propios fines. A partir de esto último, los hombres creen que es posible acceder a las cosas del entorno (a veces, Hobbes (1994) afirma incluso que, los hombres "desean una misma cosa que no puede ser disfrutada" por todos a la vez) y que todo

\footnotetext{
${ }^{3}$ De acuerdo con Hobbes, la igualdad entre los hombres (o, en rigor, la creencia de cada hombre de que todos los demás son, en el estado de naturaleza, iguales a él en términos corporales y mentales) provoca desconfianza y, posteriormente, confrontación entre ellos mismos. Pero, aceptar esto implica aceptar que, en el estado de naturaleza, a-) los recursos que se encuentran a disposición son limitados, b-) que, en tal situación, los hombres desconfían unos de otros y que, movidos ya sea por la auto-conservación o por el deleite, c-) ello los pone en situación de confrontación a los hombres. A esta altura, Hobbes no ha descrito aún el estado de naturaleza como un estado de escasez moderada, simplemente ha planteado la posibilidad de que dos hombres pueden, eventualmente, aspirar a un mismo bien y que, en tal situación, no pueden disfrutarlo simultáneamente: "Y, por tanto, si dos hombres desean una misma cosa que no puede ser disfrutada por ambos, se convierten en enemigos" (Hobbes, 1994, p. 114). El si condicional utilizado parece sugerir que tal situación es hipotética y que lo crucial es que la limitación de recursos configura un escenario de enemistad.
} 
cuanto los rodea está allí para su gozo y explotación (derecho a todo). De este modo, al concebir de ese modo el derecho natural: "Hobbes expresa una visión directamente maximizadora de la acción racional, sujeta a la condición natural, central para su psicología, que sostiene que cada individuo busca ante todo su propia preservación" (1994, p. 215).

Según se aprecia, para Gauthier, en el estado de naturaleza se describe un escenario de negociación racional en el que las partes buscan la propia preservación y el incremento del bienestar propio. Para él, el conflicto desplegado en el estado de naturaleza es de naturaleza racional (competencia) y provocado por la existencia del derecho natural. El valor está determinado por la necesidad y el juicio de otro, pues, la opinión que un hombre tiene de los demás está atravesada por la forma como ellos lo benefician y por la manera como ellos contribuyen a sus metas. Las relaciones interhumanas son, en esa medida, meras alianzas acomodaticias que hacen que en las relaciones interhumanas prime la fuerza y el fraude.

II. De acuerdo con la lectura de Gauthier, el argumento político de Hobbes requiere, en principio, que la idea de derecho no albergue ningún contenido moral $^{4}$, pues es precisamente esto lo que posibilita la emergencia del conflicto. En el Tratado del Hombre, se lee:

\begin{abstract}
Y lo que no va en contra la recta razón, todos dice que está hecho justamente y con derecho. Por el término derecho no se significa otra cosa que la libertad que todo el mundo tiene para usar de sus facultades naturales según la recta razón. Y de este modo, el primer fundamento del derecho natural consiste en que el hombre proteja, en cuanto pueda, su vida y sus miembros (Hobbes 1999, p. 18).
\end{abstract}

Gauthier encuentra en la definición transcrita dos datos claves. El primero es mencionado en El Leviatán y consiste en que lo racional es compatible con el derecho. En el estado de naturaleza cada hombre no tiene otra salida que tomar su propia razón por recta y verdadera razón (right reason). El derecho así entendido es racional mas no moral. El segundo dato clave está contenido en El Leviatán y en el Tratado del Hombre y refiere libertad. Allí, el derecho no refiere deberes sino que se trata, más bien, de una licencia, que no incluye al otro. Determina lo que el hombre puede hacer: todo, un cheque en blanco, en palabras de Gauthier. En Tratado del Hombre, se señala incluso que "en el estado de naturaleza la medida del derecho es la utilidad" (Hobbes, 1999, p. 19). Gauthier resalta el hecho de ambas definiciones implican una ausencia de contenido moral. No hay obligaciones moral derivadas de la noción de derecho natural.

\footnotetext{
${ }^{4}$ De hecho, en el estado de naturaleza no existe noción de lo justo y lo injusto: "Las nociones de lo moral y lo inmoral, de lo justo y de lo injusto no tienen allí cabida. Donde no hay un poder común, no hay ley; y donde no hay ley, no hay injusticia" (Hobbes, 1994, p. 117).
} 
Al decir de Gauthier, este derecho a todo es entendido por Hobbes como el punto de quiebre de la convivencia pacífica en el estado de naturaleza. La situación específica de la situación de guerra es consecuencia directa, precisamente, de este derecho a todo.

Y como la condición del hombre, como ya ha quedado expuesto en el capítulo precedente, es una condición de guerra de cada hombre contra cada hombre, en la que cada uno se gobierna según su propia razón y no hay nada de lo que no pueda hacer uso para ayudarse en la preservación de su vida contra sus enemigos, de ello se sigue que, en una condición así, cada hombre tiene derecho a todo, incluso a disponer del cuerpo de su propio prójimo. $\mathrm{Y}$, por tanto, mientras dure este derecho natural de cada hombre sobre cada hombre, no puede haber seguridad para ninguno, por muy fuerte o sabio que sea, ni garantía de que pueda vivir el tiempo al que los hombres están ordinariamente destinados por naturaleza (Hobbes, 1994, pp. 119-120).

Gauthier advierte que el argumento hobbesiano necesita que la guerra del estado de naturaleza no sea considerada irracional por los hombres, pero sí improductiva, inútil y poco beneficiosa. Dado que la guerra del estado de naturaleza es inútil y que ella es posible en virtud del derecho natural, Gauthier sostiene que el derecho natural es también inútil y que, motivado por ello, Hobbes introduce la idea de ley natural, en orden a plantear una limitación al derecho y a sugerir una base para la moralidad. A partir de este momento, según Gauthier, el argumento hobbesiano incorpora un elemento moral derivado de las leyes de la naturaleza, y la noción de derecho comienza a entenderse como una delimitación de lo que se debe o no se debe hacer ${ }^{5}$. La importancia de Hobbes para la teoría moral moderna halla su asiento justamente en la introducción de las leyes de la naturaleza.

\footnotetext{
${ }_{5}^{5}$ Tras la definición del concepto de derecho natural, Hobbes plantea en el capítulo 14 la existencia de dos leyes naturales que determinan la conducta humana. Con base en estas dos leyes, Hobbes describe el origen del estado político, ahonda en las circunstancias que lo preceden y da paso al estado político. Hobbes define así la primera ley fundamental de la naturaleza: "Como consecuencia, es un proceso o regla general de la razón el que cada hombre debe procurar por la paz hasta donde tenga esperanza de lograrla; y cuando no puede conseguirla, entonces puede buscar y usar todas las ventajas y ayudas de la guerra" (Hobbes, 1994, p. 120). A. P. Martinich sostiene que las dos leyes de la naturaleza evidencia un cambio significativo en la argumentación hobbesiana. El hablar de derechos, deberes y obligaciones en este contexto corrobora una transición en la argumentación hobbesiana. "Porque mientras cada hombre se aferre al derecho de hacer todo lo que le plazca, todos los hombres estarán en una situación de guerra. Pero si los hombres no renuncian a su derecho, no hay razón para que uno esté obligado a hacerlo, pues ello implicaría el convertirse en una presa para los otros, cosa que nadie está obligado" (Hobbes, 1994, p. 120). Este respeto consiste en no hacer uso efectivo del derecho a todo y en conformarse con una libertad limitada en la relación con los otros hombres. En la perspectiva de Hobbes, los hombres evalúan varios cursos de acción posibles (entre ellos perpetuar el estado de guerra de todos contra todos) y, merced a las leyes de la naturaleza, concluyen que el contrato social es el único medio hacia la preservación de la vida. Siguiendo el contenido de estas leyes, no existe cabida para una actitud diferente a: participar del acuerdo, ceder los poderes y libertades naturales y respetar lo que en el contrato se decida.
} 
Desde el punto de vista de Gauthier, el argumento hobbesiano es válido precisamente por la modificación del contenido normativo del derecho que las leyes de la naturaleza suponen. Existe así una perfecta correspondencia entre la vigencia del derecho natural y la inserción de las leyes de la naturaleza. Establecer un derecho es determinar lo que es bueno y lo que es malo, lo que se puede hacer y lo que no. Por esta razón, las leyes de la naturaleza distan de ser meros consejos. Difieren de ellos en razón a su generalidad y su necesidad, pues establecen, primero, lo que se debe hacer para asegurar un fin, la preservación, y, segundo, lo que todos deben hacer para asegurar un fin: la autoconservación.

La inserción de las leyes de la naturaleza en el argumento hobbesiano frena, pues, la injerencia del derecho ilimitado a todas las cosas y posibilita el tránsito de una moral natural/individual a una moral convencional/grupal. Gauthier entiende este concepto de la siguiente manera:

Definiré una convención como una regularidad $\mathrm{R}$ en el comportamiento de personas $\mathrm{P}$ en una situación $\mathrm{S}$, tal que parte de la razón para que la mayoría de estas personas actúen en conformidad con $\mathrm{S}$ es la idea común de que la mayoría de personas actúan o actuarán en conformidad con R en S, y que la mayoría espera que los demás actúen R en S (1979, p. 552).

Así, una convención es una regularidad que todos esperan que se dé y que, en esa medida, induce a los hombres a actuar de un modo compatible con ella. ${ }^{6}$ Llevando esto al asunto hobbesiano, Gauthier anota:

If the rationale for adherence to them is to rest on the knowledge that adherence is both usual and expected, then two conditions must be satisfied. First, each person must have reason to prefer that most persons adhere to the laws of nature, rather than that most ignore the laws; otherwise the convention would be pointless for those who lacked such reason. And second, each person must have reason to prefer that he or she ignore the laws of nature, given that most others ignore them; otherwise the convention would be redundant, since each would have reason to adhere whether others did so or not. The laws of nature are not pointless, since mutual adherence to them is necessary to bring men from a condition of war to one of peace. And they are not redundant as conventions, since, as Hobbes insists, no one has reason to adhere to them unless others do $(1979$, p. 552).

\footnotetext{
${ }^{6}$ Gauthier indica que existen dos tipos de convención: la descriptiva (descripción) y la perspectiva (justificación). La de Hobbes es una convención prescriptiva, toda vez que las leyes de la naturaleza sugieren un tipo de comportamiento. A partir de ellas, se espera que los hombres se comporten de un determinado modo.
} 
El contenido moral de las leyes de la naturaleza constituye de este modo un conjunto de convenciones comportamiento (regulare) orientado a la consecución de la paz. Los valores son subjetivos, pero la paz es un bien (esto es, un valor) instrumental común. Las leyes son racionales para todos, en tanto que son condición de posibilidad de la paz. Lo decisivo es así el tránsito del estado de naturaleza al convencional, determinado éste por la modificación de la naturaleza de la razón: del instrumentalismo al convencionalismo.

Reason is instrumental, but the laws of nature, which prescribe the means of peace, are addressed equally to each man's reason, and so are rational for all. Interest is non-tuistic, yet each man must give up some of the right with which he pursues his own interests, since this is the basis of the laws of nature. Thus morality, a set of conventions constraining each man's maximizing activity, and distinguishing right from wrong, is established. (Gauthier, 1979, p. 553)

III. Y bien, ¿qué tan estables son el conjunto de convenciones que se derivan de la moralidad hobbesiana y de las leyes de la naturaleza? En otras palabras, ¿de qué manera son las convenciones estables? Gauthier opina que son racionalmente estables si cada persona tiene razones para adherirse a ellas y espera que los demás también las tengan. Son motivacionalmente estables si usualmente cada cual se siente movido a actuar de una manera específica. Para Gauthier, los autores contractualistas conciben y resuelven el problema racional de la estabilidad, pero descuidan el problema motivacional. A su parecer, Hobbes es el único que se ocupa también de este problema analizando el comportamiento de lo que él denomina como necio o insensato.

El insensato dice en su corazón que no existe tal cosa como la justicia; y a veces lo dice también con su lengua. Y alega, con toda seriedad, que, como la conservación y la felicidad de cada hombre está encomendada al cuidado que cada cual tiene de sí mismo, no puede haber razón que impida a cada uno hacer todo lo que crea que puede conducirlo a alcanzar esos fines. Y así, hacer o no hacer convenios, cumplirlos o no cumplirlos, no es proceder contra razón, si ello redunda en beneficio propio. El insensato no niega, ciertamente, que haya convenios, y que éstos son unas veces respetados, y otras no, y que su incumplimiento puede llamarse injusticia, y que su observancia es sinónimo de justicia; pero se hace todavía cuestión de si la injusticia[...] no podrá a veces ser compatible con esa razón que dicta a cada uno buscar su propio bien, particularmente cuando conduce a un beneficio tal que no sólo pone a un hombre en situación de despreciar los ultrajes y reproches de otros hombres, sino también el poder de éstos (Hobbes, 1994, p. 132). 
Según Gauthier, el problema racional planteado por el caso del insensato se resuelve mediante la racionalidad de la justicia. En el insensato la niega porque considera que el beneficio de la violación unilateral del pacto es, en todos los escenarios posibles, superior a la observancia de los términos del pacto y de la adhesión mutua. El insensato considera que lo más racional es la ventaja anticipatoria, el comportamiento bélico, expresado en la violación unilateral del pacto.

Since mutual anticipation creates war, Hobbes holds that it is rational for each person to lay down the right to anticipate, provided others do so as well. But however true this may be, it does not change the advantage inherent in anticipation, which still maximizes each person's prospect for survival and so is rational. If violating one's covenant enables one to anticipate one's fellows, then it is rational. Hence, if the rational man seems to lay down some portion of his right, it can only be to take it up again as the occasion may suggest. But then morality is indeed in vain. Each may pretend peace, but only the better to anticipate his fellows (Gauthier, 1979, p. 554).

La respuesta de Hobbes al caso del insensato es que él será excluido de la sociedad por parte de los otros hombres que piensan que la observancia del pacto sí genera mayores beneficios. Según Hobbes, es racionalmente esperable conductas como la del insensato, pero no es racionalmente esperable que los otros hombres permitan al insensato vivir en paz en el interior de la sociedad. Él siempre será excluido, pues, es lo más y único racional. En relación con el pacto, Gauthier describe los tres posibles escenarios que se siguen de la argumentación de Hobbes:

1. Que la adhesión mutua puede ser mejor que la violación unilateral.

2. Que la adhesión mutua puede ser peor que la violación unilateral, pero mejor en virtud del esfuerzo colectivo.

3. Que la adhesión mutua puede ser peor que la violación unilateral en algunos momentos. (Hobbes citado por Gautier, 1979).

Conforme se ha expuesto, Hobbes sugiere que es mejor observar el acuerdo que violarlo. Ese es el núcleo de su respuesta al insensato. Hobbes parte del supuesto que el único escenario que los hombres constituirán es el primero. El insensato sostiene que 2 es mejor que 1 y 3 . Por su parte, Hobbes afirma que 1 es la opción más racional: esto es, una limitación a la ventaja maximizadora de la utilidad (leyes de naturaleza). El supuesto de Hobbes es que la violación unilateral genera beneficios menores a la ganancia de la adhesión mutua y el esfuerzo colectivo. Ahora, pese a que Hobbes considera que ha dado respuesta a la objeción del insensato, Gauthier opina que esta solución es débil e insuficiente, y por ello ofrece una distinta, que nada tiene que ver con el supuesto del que parte Hobbes. Según Gauthier, cuando se niega a la cooperación mutua, el insensato apela a un concepto de razón propio del estado de naturaleza. Su razón es natural 
no recta (recta razón), de suerte que la no comprensión de la relación entre razón y derecho lleva al insensato a violar unilateralmente el pacto. Pues, renunciar al derecho natural a todo implica renunciar a acudir a la razón natural; renunciar al derecho natural implica renunciar a la guerra y aceptar la búsqueda de la paz. En opinión de Gauthier, el insensato es, de este modo, contradictorio porque acude a algo a lo cual renunció.

The right of nature expresses right reason. If one lays down some portion of that right, then one also renounces the rationality that was the basis of the right laid down. If one lays down some portion of one's right to do whatever seems conducive to one's preservation and well-being, so that one may find peace, then one renounces preservation as the standard of reason, in favor of peace. The Foole appeals to that reason which dictates to every man his own good-to natural reason, so that he may show injustice to be rational. But injustice is a violation of covenant, and, in covenanting, in laying down one's right, one has renounced natural reason as the court of appeal, in favor of a reason that dictates to every man what all agree is good (1979, p. 557).

IV. En el análisis presentado, Gauthier sostiene que la razón subjetivamente entendida (derivada del derecho natural y proclive al error) conduce a una situación de guerra de todos contra todos. Desde su punto de vista, la recta razón presupone una moralidad que contiene un árbitro de las disputas y un conjunto de comportamientos convencionales sugerido por las leyes de la naturaleza y vigilado por el soberano. Según esta lectura, la clave se encuentra en la distinción atribuida a Hobbes entre razón natural y razón convencional. Mediante esto, para Gauthier, el contrato social de Hobbes, mecanismo que compatibiliza las expectativas individuales y colectivas, resuelve tanto el problema racional (fundamentación) como el motivacional (estabilidad). El primero mediante la aclaración acerca de la proclividad al error de la razón natural y la necesidad de un árbitro (que dirima disputas y evite la guerra) y el segundo mediante un soberano (que ponga coto a las pretensiones de los eventuales violadores unilaterales de los pactos).

The problem of motivational stability is, of course, not resolved by replacing natural with conventional reason. We may grant the Foole that each person would prefer to violate the laws of nature, given that others adhere. Since men tend to be ruled by passion rather than reason, Hobbes requires the Sovereign, not only as arbiter, whose reason, accepted by all as right reason, prescribes the means to peace, but also as enforcer, whose power, authorized by all, is exercised to maintain peace. But this problem of motivation is not peculiar to Hobbes' conception of morality and does not threaten to undermine his conventionalist theory (Gauthier, 1979, p. 558). 
En este sentido, Gauthier considera que el soberano resuelve los problemas iniciales, presente, y los posteriores, futuro ${ }^{7}$. En el presente, el problema es la guerra, cuya solución es la institución del soberano que pone coto a las pretensiones auto-interesadas. En el futuro, el problema consistirá en lidiar con los freeriders, los insensatos y todos aquellos que amenacen la estabilidad del régimen político. Así, el objetivo inicial es la paz y el posterior es la estabilidad. A diferencia de otros autores, Gauthier considera que Hobbes integra a su propuesta circunstancias ulteriores al contrato. Su perspectiva del conflicto interhumano es, en ese sentido, más adecuada que otras que sólo consideran las disputas contempladas en el estado de naturaleza y que parecen ignorar las que el estado político trae consigo (Kavka, 1983) $\Phi$

\section{REFERENCIAS}

Gauthier, D. (1969). The Logic of "Leviathan": The Moral and Political Theory of Thomas Hobbes. London: Oxford at Clarendon Press.

Gauthier, D. (1979). "Thomas Hobbes: Moral Theorist". The Journal of Philosophy, 76(10), pp. 547-559.

Gauthier, D. (1987). Morals by Agreement. London: Oxford at Clarendon Press.

Hobbes, Th. (1994). Leviatán: La materia, forma y poder de un Estado eclesiástico y civil. Madrid: Alianza.

Hobbes, Th. (1999). Tratado del Hombre. Madrid: Trotta.

Kavka, G. (1983). Hobbes's War of All Against All. Source: Ethics. 93(2), pp. 291310.

\footnotetext{
En la reconstrucción que hace Gauthier del argumento hobbesiano, el soberano tiene dos tareas fundamentales: 1-) atemorizar y 2-) coordinar los esfuerzos individuales de modo que redunden en beneficio del colectivo. Con objeto explicar cómo 1-) es condición de posibilidad de 2-), Gauthier detalla dos partes en el procedimiento utilizado por Hobbes: 1-) el convenio y 2-) la autorización. A su juicio, 2-) provee el contenido de 1-). Gauthier afirma que, para Hobbes, un convenio (covenant) es un tipo de contrato (contract), difiriendo con ello de Hampton quien considera el empleo de esta terminología es equivocado (misleading).
} 